American Journal of Pharmaceutical Education 2021; 85 (5) Article 8259.

\title{
BRIEF
}

\section{Impact of a Simulated Legislative Visit on Student Pharmacists' Political Skill Inventory Scores}

\author{
Cortney M. Mospan, PharmD ${ }^{\mathrm{a}}$, Chris Gillette, $\mathrm{PhD}^{\mathrm{b}}$ \\ ${ }^{a}$ Wingate University, School of Pharmacy, Wingate, North Carolina \\ ${ }^{\mathrm{b}}$ Wake Forest University, School of Medicine, Winston-Salem, North Carolina \\ Submitted June 29, 2020; accepted December 22, 2020; published May 2021.
}

Objective. To assess the impact of a simulated legislative visit on the Political Skill Inventory (PSI) scores of Doctor of Pharmacy (PharmD) students and assess their perceptions of their role as an advocate.

Methods. Anonymous pre- and post-intervention surveys were administered online to third year student pharmacists at one school of pharmacy in North Carolina. Measures included gender, age, previous pharmacy experience, engagement in pharmacy organizations, legislative awareness and engagement, political skill inventory, and perceptions of the simulated legislative visit. Data are presented as medians or proportions, where appropriate. Paired t tests and Cohen $d$ were used to analyze the data.

Results. Thirty student pharmacists provided complete and matchable results for the pre- and post-intervention surveys (analysis response rate $=36 \%$ ). The mean PSI score was 5.4 before the intervention and increased to 5.7 after the intervention. The networking ability and interpersonal influence subscales showed significant and medium-to-large increases in effect size whereas the social astuteness and apparent sincerity subscales showed low-to-medium increases in effect size. Student pharmacists' agreement with advocacy skills or responsibilities increased overall from pre-to post-intervention.

Conclusion. The political skill inventory scores of student pharmacists significantly improved following participation in a simulated legislative visit and listening to a corresponding advocacy lecture. Determining the effectiveness of educational interventions to develop advocacy skills in student pharmacists is essential to ensure efficacy and potential for PharmD students and graduates to make real-world impacts.

Keywords: advocacy, student pharmacist, political skill inventory, legislation

\section{INTRODUCTION}

At an American Association of Colleges of Pharmacy (AACP) Curricular Change Summit in 2009, five key abilities for student pharmacists that needed to be addressed in pharmacy curricula of the future were identified. Among these, leadership and advocacy were identified as being perhaps the most important. ${ }^{1}$ Despite this emphasis, the majority of advocacy experiences described in the pharmacy education literature are cocurricular or elective didactic coursework and often facilitated by student organizations of state or national pharmacy organizations. ${ }^{2-7}$ While these are valuable experiences for student pharmacists, there are inherent issues with these delivery models, such as capacity limitations, inaccessibility that may occur because of financial costs and potential need for excused absences from other curricular responsibilities.

Corresponding Author: Cortney M. Mospan, Wingate University, School of Pharmacy, 515 N Main St., Wingate, NC 28174. Tel: 704-233-8329. Email: c.mospan@

wingate.edu
In the limited studies describing advocacy training in core pharmacy curricula, the outcomes primarily focus on student pharmacists' performance on course activities or student pharmacist perceptions, which may not predict true growth or a student's ability to perform advocacy skills. Outcomes from elective courses often describe student pharmacists' learning how to access information, registering to vote, engaging in legislative experiences, and improving their self-reported advocacy-related skills. ${ }^{2,4}$ Regardless of the delivery method for advocacy curricula, whether via the core didactic curriculum, elective didactic curriculum, or co-curricular experiences, there is a lack of assessment of improvement as a result of these curricular interventions in student pharmacists' legislative or political advocacy ability using a validated tool.

The Political Skill Inventory (PSI), created by Ferris and colleagues, is a validated, reliable, and stable construct to assess a person's political skill. ${ }^{10-12}$ High scores on the PSI have been positively associated with political savvy and emotional intelligence, and a predictor of the subject's job performance and work-related behaviors 


\section{American Journal of Pharmaceutical Education 2021; 85 (5) Article 8259.}

and outcomes. ${ }^{10,11}$ Further, political skill represents the ability to maximize and leverage relationships in order to achieve goals at the personal, team, and organization level. ${ }^{13}$ These are necessary skills for engaging in political advocacy. While simulated or mock legislative visits have been used previously to assess student pharmacists' advocacy skills and prepare them to use the skills in the future, these interventions were not assessed using validated tools or assessment methods. ${ }^{5,14}$ The objectives of this study were to assess the impact of a simulated legislative visit on student pharmacists' PSI scores and assess student pharmacists' perceptions of their role as an advocate. The authors hypothesized that a simulated legislative visit following a lecture on developing a "legislative ask" (a proposal to ask to vote in support of a mock bill after with a pitch for the bill) would lead to improved PSI scores.

\section{METHODS}

This study was deemed exempt by the Wingate University Research Review Board (IRB). In January 2019, an anonymous online pre- and post-intervention surveys were administered to third professional year (P3) student pharmacists $(n=83)$ enrolled in the Current Issues in Public Health course. Student pharmacists were included in the study analysis if they provided usable survey responses for both the pre- and post-intervention survey. Informed consent was obtained from students at the beginning of the pre-intervention survey. The surveys were administered using Qualtrics XM (Qualtrics, LLC). This study was designed to assess student pharmacists' PSI scores at baseline and after attending a lecture, developing a legislative ask, and completing a simulated legislative visit.

The lecture was developed using two resources: an article by Gohlke and colleagues entitled "Igniting the Fire Within: A Primer on Political Advocacy for Pharmacy Professions," and a book by Boyle and colleagues entitled Leadership and Advocacy for Pharmacy. ${ }^{15,16}$ The lecture objectives, the simulated legislative visit checklist, and a summary of the strategies for developing a legislative ask mapped to the PSI are presented in Figure 1. These strategies were developed from the first author's legislative experiences and the previously cited resources. Student pharmacists were provided with a piece of mock legislation in advance of the simulated legislative visit and a case description for a pharmacy legislative day to enable them to prepare their legislative ask for the mock legislator. Student pharmacists were tasked with advocating for the mock legislation, which would allow pharmacists to provide smoking cessation treatment for patients via a statewide standing order, and asking the mock legislator to vote in support of the legislation. The students' performance during the simulated legislative visit was assessed using a rubric that was mapped to the simulated visit checklist (Figure 1).

The following student pharmacist demographic characteristics were collected on the pre-intervention survey: gender identification, previous pharmacy work experience, and type of pharmacy work experience. We collected information on the students' work experience because pre-matriculation work experience has been shown to influence development of professional identity in student pharmacists as well as perceptions about their future career. ${ }^{17-19}$ Student pharmacists were also asked how many organizations they were a dues-paying member of, their knowledge about their elected legislators, their engagement in the legislative process, and their attitudes and self-perceived skills regarding advocacy roles and responsibilities.

We identified two validated tools to assess students' advocacy-related skills, interest, and activities: the PSA and the Political Astuteness Inventory (PAI). The PSI measures the ability to read situations and people, whereas the PAI is designed to measure awareness and understanding of legislative and political processes (ie, voting activity). ${ }^{10,20}$ Because the goal of this project was to assess the impact of a simulated legislative visit where student pharmacists were tasked to try to garner the support of a mock legislator, it was deemed that the PSI was a better fit than the PAI. Thus, all study participants were asked to complete the PSI.

The 18-item PSI is designed to measure political skill and consists of the following dimensions: networking ability, interpersonal influence, social astuteness, and apparent sincerity. Each item is scored using a sevenpoint Likert-type scale, with answers ranging from $1=$ strongly disagree to $7=$ strongly agree.$^{10}$

For the PSI, the subject receives a total score, which is then divided by 18 (number of items in the inventory) to arrive at a mean PSI composite score and a score for each subscale. A higher mean score $(>4.0)$ denotes greater than average political skill. The internal consistency of the PSI has been reported to be $\alpha=0.89-0.96$, indicating adequate internal consistency. ${ }^{10,21,22}$ On the pre-intervention survey, students' attitudes and skills were assessed and the PSI was completed. On the post-intervention survey, student pharmacists completed the PSI a second time and were asked their perceptions of the simulated legislative visit.

All analyses were conducted using SAS, version 9.4 (SAS Institute). Pre- and post-intervention datasets were compared to the matched dataset to determine whether 
American Journal of Pharmaceutical Education 2021; 85 (5) Article 8259.

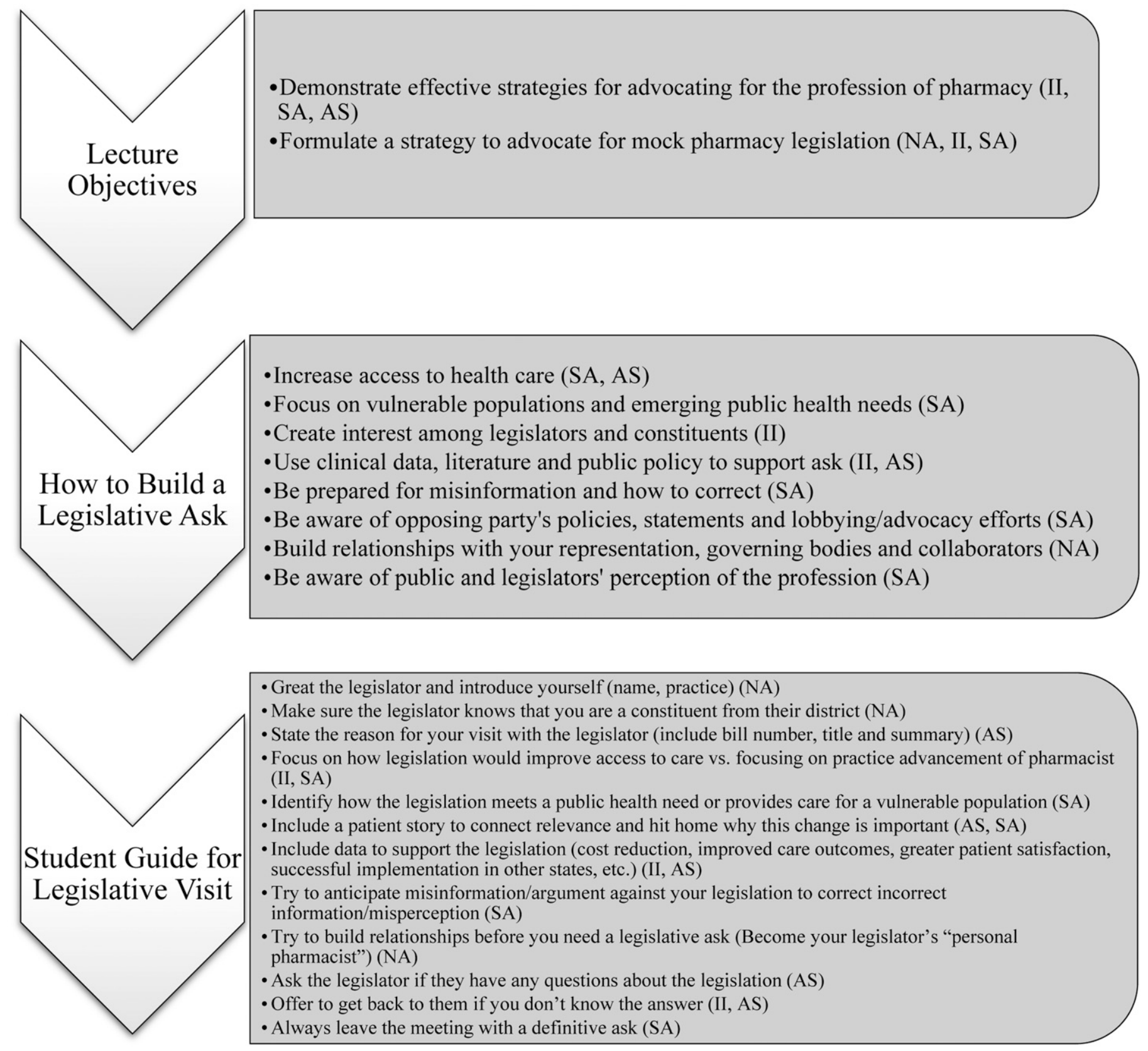

$\mathrm{NA}=$ networking ability, $\mathrm{II}=$ interpersonal influence, $\mathrm{SA}=$ social astuteness, $\mathrm{AS}=$ apparent sincerity

Figure 1. Lecture Objectives, Course Lecture, and Guide for Legislative Visit Mapped to the Political Skill Inventory

having a lower sample size resulted in any significant differences that might have biased our interpretation of the results. For the total PSI scale and each subscale on both the pre- and post-intervention survey, one-sample $t$ tests were used to compare whether the mean for the postintervention survey was significantly different from the pre-intervention survey using an alpha less than $p=.05$. No significant differences were found between the preand post-intervention responses of students in the matched sample, so the decision was made to only use the matched sample for data analysis.

Descriptive statistics were utilized for demographics. Internal consistency of the PSI was assessed using Cronbach alpha. The PSI scores were reported using means and standard deviations (SDs). To account for the possibility of a type I error due to multiplicity of testing for each of the subscales, the Bonferroni correction $(\alpha / 5)$ was used to adjust the alpha to $p<.01$ to indicate statistical 


\section{American Journal of Pharmaceutical Education 2021; 85 (5) Article 8259.}

significance. Bivariate relationships were assessed between the following variables and the pre-test PSI average score using an independent samples t test or one-way analysis of variance (ANOVA), when appropriate: gender, age group, previous degrees, and number of pharmacy organizations in which the student pharmacist was a dues paying member. Change in PSI average score and each of the PSI subscales were assessed using paired $t$ tests and Cohen $d$. All statistical tests were two-sided. Increases in attitudes regarding advocacy roles and responsibilities were assessed from pre- to post-intervention. To preserve statistical power, no bivariate statistical analyses were used. Student pharmacist attitudes regarding pharmacist advocacy are reported after participating in the simulated legislation visit using descriptive statistics (frequencies and proportions).

\section{RESULTS}

Sixty-four student pharmacists completed the preintervention survey (initial response rate $=77 \%$ ) and 44 student pharmacists completed the post-intervention survey (follow-up response rate $=53 \%$ ). Complete and usable matched data were obtained for 30 student pharmacists (analysis response rate $=36 \%$ ). The majority of the sample were female $(77 \%, \mathrm{n}=23)$, from 20 to 25 years old $(73 \%, \mathrm{n}=22)$, did not have a prior degree $(53 \%$, $\mathrm{n}=16$ ), and had previous paid pharmacy work experience $(93 \%, n=28)$. The most common location for previous paid pharmacy work experience was in a chain community pharmacy $(63 \%, \mathrm{n}=19)$. Most student pharmacists $(80 \%, n=24)$ were not aware of who their state legislators were and $77 \%(n=23)$ were not aware of who their federal legislators were. Most student pharmacists had never contacted their legislators about pharmacy-specific legislation $(73 \%, \mathrm{n}=22)$ nor non-pharmacy specific legislation $(87 \%, \mathrm{n}=26)$.

The pre-survey PSI internal consistency was $\alpha=.92$ while the post-test PSI internal consistency was $\alpha=.94$. For the PSI total scale and each subscale, student pharmacists reported greater than average (mean $>4.0)$ political skill. The mean for the total PSI scale and each subscale pre- and post-intervention are given in Table 1. At baseline, there were no significant differences on the mean PSI score based on any of the student pharmacist demographic characteristics $(p>.05)$. The paired $t$ test indicated that, on average, student pharmacists composite PSI score had significantly increased $(\mathrm{t}=4.35, \mathrm{DF}=29, p<.001, d=.79)$ from pre- to post-intervention. The paired t tests for the networking ability subscale $(\mathrm{t}=4.07, \mathrm{DF}=29, p<.001$, $d=.74)$ and the interpersonal influence subscale $(\mathrm{t}=3.52$, $\mathrm{DF}=29, p<.005, d=.64)$ indicated that mean scores had significantly increased, with a medium to large effect size. However, the paired $\mathrm{t}$ tests for the social astuteness subscale $(\mathrm{t}=2.25, \mathrm{DF}=29, p=.03, d=.41)$ and apparent sincerity subscale $(\mathrm{t}=2.44, \mathrm{DF}=29, p=.02, d=.45)$ did not increase significantly. Even though the $p$ values did not reach the critical threshold for significance for the social astuteness and apparent sincerity subscales, there were small to medium effect size increases indicated by Cohen $d$.

None of the student pharmacist demographic characteristics were found to be associated with the change in PSI score $(p>.05)$. For each item regarding advocacy skills or responsibilities, the proportion of student pharmacists who agreed or strongly agreed increased from pre- to post-intervention for all items except one. There was a decrease in the percentage of student pharmacists reporting they agreed or strongly agreed that it was part of their role as a student pharmacist to advocate for the health needs of society from $97 \%(n=29)$ to $87 \%(n=26)$. The percent change for each item is shown in Table 2.

Regarding student pharmacist attitudes toward the simulated legislation visit, $90 \%(n=27)$ indicated that they agreed or strongly agreed that the simulated legislative visit was effective at improving their confidence when participating in advocacy. Ninety-three percent $(n=28)$ of student pharmacists indicated that they agreed or strongly agreed that participating in the simulated

Table 1. Political Skills Inventory Scores Before and After Pharmacy Students Participated in a Simulated Legislative Visit $(\mathrm{N}=30)$

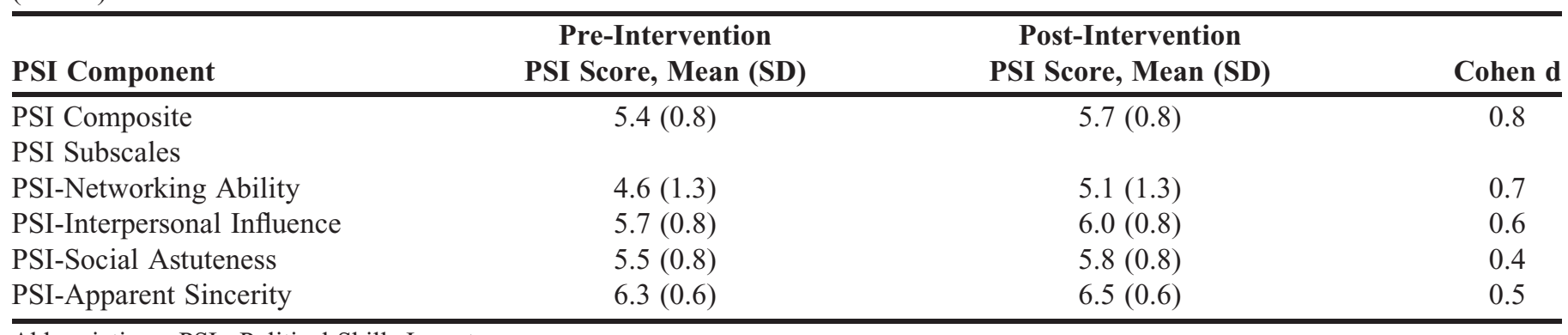

Abbreviations: PSI=Political Skills Inventory 


\section{American Journal of Pharmaceutical Education 2021; 85 (5) Article 8259.}

Table 2. Student Pharmacists' Perceptions of Advocacy Skills and Responsibilities Before and After Participating in a Simulated Legislative Visit $(n=30)$

\section{Statement}

\begin{tabular}{cc}
$\begin{array}{c}\text { Pre-Intervention, } \\
\text { Strongly Agree } \\
\text { or Agree, \% }\end{array}$ & $\begin{array}{c}\text { Post-Intervention, } \\
\text { Strongly Agree } \\
\text { or Agree, \% }\end{array}$ \\
\hline 43 & 90 \\
13 & 77 \\
40 & 97 \\
67 & 73 \\
70 & 73 \\
83 & 93 \\
60 & 73 \\
43 & 60 \\
40 & 77 \\
97 & 87 \\
33 & 53 \\
\hline
\end{tabular}

legislative visit better prepared them for engaging as an advocate. Finally, 67\% $(n=20)$ of student pharmacists indicated that they agreed or strongly agreed that they were more likely to engage in advocacy activities as a student pharmacist or pharmacist after participating in the simulated visit.

\section{DISCUSSION}

Despite a greater than average composite baseline PSI score, third year student pharmacists showed significant improvement in composite PSI scores as well as in networking ability and interpersonal influence subscale PSI scores following preparation of a legislative ask and completion of a simulated legislative visit. While the social astuteness and apparent sincerity subscale scores did not change significantly, they also showed improvement. The PSI showed high internal consistency among the study population that was similar to that reported among a population of nurses. ${ }^{22}$ To the authors' knowledge, this is the first study to evaluate the impact of advocacy training among a student pharmacist population using a validated tool, the PSI. Student pharmacists in previous studies also reported improvement in their advocacy-related attitudes and skills; however, people have a tendency to overestimate their competence. ${ }^{8,23-25}$

Student pharmacists in this study reported that the simulated legislative visit was effective in preparing them for advocacy activities and that they were more likely to participate in advocacy initiatives following the experience. This is important as Gang and colleagues identified a self- reported duty to advocate among health profession students that did not translate to student knowing how to advocate or taking action. This could have been impacted by a very low prevalence of advocacy training. ${ }^{26}$ This low prevalence of advocacy training has led some health systems to create interprofessional health policy and advocacy curricula as part of postgraduate training. ${ }^{23}$ There have been several calls for mandatory "hands on" or skill-based curricula in advocacy in medical school programs. ${ }^{25-27}$ Ross and colleagues reported that "hands on" learning activities were available at $11 \%-57 \%$ of US schools and colleges of pharmacy, with the most common activity being participation in a legislative day. This is similar to the activities used in medical education where approximately $50 \%$ of institutions provide opportunities for students to practice advocacy. ${ }^{3}$

This study had several limitations that must be considered. The study sample size was small, which may have contributed to a type II error for the social astuteness subscale. Other possible causes of a type II error in this subscale was the use of the Bonferroni correction. Further, the study population represented one class of student pharmacists at one academic institution. Before the PSI could be recommended as a tool to assess the development of advocacy-related skills among student pharmacists, this study would need to be replicated at multiple pharmacy schools and have a larger sample size. The findings of this study suggest that the PSI may be a reliable tool to assess political skills in student pharmacists. Additionally, the post-intervention assessment occurred directly after the simulated legislative visit, and no long-term 


\section{American Journal of Pharmaceutical Education 2021; 85 (5) Article 8259.}

assessment of retention of skills was conducted. The study institution has a longitudinal curricular thread in advocacy similar to the thread described by Mospan and colleagues; however, in their study the baseline assessment occurred midway through student pharmacists' exposure to the thread. ${ }^{30}$

Future research will focus on assessment of PSI scores from the beginning to the end of the didactic PharmD curriculum at the study institution to assess the impact of a longitudinal advocacy curricular thread. Martin and colleagues have highlighted the importance of the lack of clarity in how to assess advocacy skills and have called for assessments beyond pre-post assessment that show meaningful long-term impact through learner engagement in advocacy. ${ }^{31}$ This is necessary before the results can be extrapolated to other PharmD curricula given the higher than average baseline PSI score. Despite these score differences, internal consistency was shown to be comparable to that of previous studies. ${ }^{10,21,22}$ Student pharmacists had already been exposed to a portion of the advocacy curricular thread, which could explain the higher baseline PSI score than found in other studies. Additionally, skills that the PSI measures may be skills that are difficult to teach and are instead skills that are more determined by innate personality traits. Replication of these results at other institutions that offer simulated legislative visits or legislative days could help to establish that PSI skills can be taught in the PharmD curricula and enhanced with proper advocacy training.

\section{CONCLUSION}

The PSI was shown to have high internal consistency for evaluating the impact of a simulated legislative visit on a cohort of third-year student pharmacists. Although improvement associated with a simulated legislative visit was seen in the students' PSI scores, significant improvement was only seen in scores on the networking ability and interpersonal influence subscales. The Political Skill Inventory and the simulated legislative visit show promise as an effective assessment tool and core didactic teaching tool, respectively, for advocacy training in student pharmacists. Future research is needed to validate these findings among a larger sample size of student pharmacists at multiple institutions who receive different advocacy-related curricular experiences.

\section{REFERENCES}

1. Jungnickel PW, Kelley KW, Hammer DR, Haines ST, Marlowe K. Addressing competencies for the future in the professional curriculum. Am J Pharm Educ. 2009;73(8):158.

2. Blake EW, Powell PH. A pharmacy political advocacy elective course. Am J Pharm Educ. 2011;75(7):137.
3. Ross LA, Janke KK, Boyle CJ, et al. Preparation of faculty members and students to be citizen leaders and pharmacy advocates. Am J Pharm Educ. 2013;77(10):220.

4. Pace AC, Flowers SK. Students' perception of professional advocacy following a political advocacy course. Curr Pharm Teach Learn. 2012;4(1):34-38.

5. Adams AJ, Matzke GR, McCall KL. A novel education and training program to enhance student advocacy. Am J Pharm Educ. 2015;79(7):95.

6. Fritsch MA, Culver N, Culhane N, Thigpen J, Lin A. AdvoCaring: a cocurricular program to provide advocacy and caring to underserved populations in Baltimore. Am J Pharm Educ. 2016; 80(7): 126 .

7. Deloatch KH, Gannett PM, Hagemann TM, et al. Student engagement in professional political advocacy in colleges/schools of pharmacy. Curr Pharm Teach Learn. 2012;4(3):207-211.

8. Mospan CM, Mospan GA. Assessing the impact of a didactic lecture for student pharmacists on legislative advocacy. Curr Pharm Teach Learn. 2018;10(10):1363-1374.

9. Mraiche F, Paravattil B, Wilby KJ. The use of oral presentations, role-play sessions, and reflective critiques to emphasize advocate learning outcome in the pharmacy curriculum. Curr Pharm Teach Learn. 2015;7(4):443-450.

10. Ferris GR, Treadway DC, Kolodinsky RW, et al. Development and validation of the political skill inventory. J Manag. 2005;31(1): 126-152.

11. Jacobson RK, Viswesvaran C. A reliability generalization study of the political skill inventory. SAGE Open. 2017;7(2):1-17.

12. Lvina E, Johns G, Treadway DC. Measure invariance of the Political Skill Inventory (PSI) across five cultures. Int J Cross Cult Manag. 2012;12(2):171-191.

13. Braddy P, Campbell M. Using political skill to maximize and leverage work relationships. Center for Creative Leadership website. https://cclinnovation.org/wp-content/uploads/2020/03/ usingpoliticalskill.pdf. Accessed April 27, 2021.

14. Johnson C, Traynor A. Using visits with simulated legislators to improve pharmacy students' advocacy skills. Am J Pharm Educ. 2016;80(5):S2:92.

15. Gohlke AL, Murphy KM, Cannell ME, Ray DB, Burnworth MJ. Igniting the fire within: a primer on political advocacy for pharmacy professionals. J Pharm Pract. 2013;26(3):165-170.

16. Boyle CJ, Beardsley RS, Matzke GR. Leadership and Advocacy for Pharmacy, 2nd ed. American Pharmacists Association; 2014.

17. Bloom TJ, Smith JD, Rich W. Impact of pre-pharmacy work experience on development of identify in student pharmacists. Am J Pharm Educ. 2017;81(10):6141.

18. Siracuse VM, Schondelmeyer SW, Hadsall RS, Schommer JC. Third-year pharmacy students' work experience and attitudes and perceptions of the pharmacy profession. Am J Pharm Educ. 2008; 72(3):50.

19. Siracuse MW, Clark BE. Third-year pharmacy students' work experiences and attitudes. Innov Pharm. 2017;8(2):16.

20. Clark PE. Political astuteness inventory. In Clark PE, ed. Community assessment reference guide for community health nursing. 1st ed. Pearson Prentice Hall; 2008.

21. Coole DR. Expansion and validation of the political skill inventory (PSI): An examination of the link between charisma, political skill, and performance. Graduate Theses and Dissertations. 2007. http://scholarcommons.usf.edu/etd/680. Accessed April 27, 2021. 


\section{American Journal of Pharmaceutical Education 2021; 85 (5) Article 8259.}

22. Montalvo W, Byrne MW. Mentoring nurses in political skill to navigate organizational politics. Nurs Res Pract. 2016;2016:

3975634.

23. Long T, Chaiyachati KH, Khan A, Siddharthan T, Meyer E, Brienza R. Expanding health policy and advocacy education for graduate trainees. J Grad Med Educ. 2014;6(3):547-550.

24. Huntoon KM, McCluney CJ, Wiley EA, Scannell CA, Bruno R, Stull MJ. Self-reported evaluation of competencies and attitudes by physicians-in-training before and after a single day legislative advocacy experience. BMC Med Educ. 2012;12:47.

25. DeAngelis T. Why we overestimate our competence. Monitor on Psychology. 2003;34(2):60.

26. Gang M, Tseng Z, Baird GL, Egan P, McGarry K. Attitudes toward advocacy do not match actions: a cross-sectional survey of residents and fellows. $R$ I Med J (2013). 2019;102(3):34-37.
27. Douglas A, Mak D, Bulsara C, Macey D, Samarawickrema I. The teaching and learning of health advocacy in an Australian medical school. Int J Med Educ. 2018;9:26-34.

28. Bhate TD, Loh LC. Building a generation of physician advocates: the case for including mandatory training in advocacy in Canadian medical school curricula. Acad Med. 2015;90(12): 1602-1606.

29. Zaidi D, Lichstein PR. Advocacy may have a place in medical curricula. N C Med J. 2019;80(6):383.

30. Mospan CM, Calhoun M. Developing students as advocate through a pilot advocacy curricular thread within a PharmD curriculum. Innov Pharm. 2016;7(3):17.

31. Martin D, Hum S, Han M, Whitehead C. Laying the foundation: teaching policy and advocacy to medical trainees. Med Teach. 2013; 35(5):352-358. 